\title{
Polymorphisms in rs915941 and rs915942: Are they associated with increased risk of G6PD enzyme deficiency in the Sri Lankan population?
}

\author{
R Dewasurendra ${ }^{1 *}$, N Sepulveda ${ }^{2}$, N Chandrasekharan ${ }^{3}$, N Karunaweera $^{1}$ and S Gunawardena ${ }^{1}$ \\ ${ }^{1}$ Department of Parasitology, Faculty of Medicine, University of Colombo, 25, Kynsey Road, Colombo 08. \\ ${ }^{2}$ Department of Infection Biology, Faculty of Infectious and Tropical Diseases, London School of Hygiene and Tropical Medicine, London, UK. \\ ${ }^{3}$ Department of Chemistry, Faculty of Science, University of Colombo, Colombo 03.
}

\begin{abstract}
Radical treatment of imported malaria is vital for prevention of re-introduction of the disease in Sri Lanka. Individuals with glucose-6-phosphate dehydrogenase (G6PD) enzyme deficiency are subject to haemolytic anaemia with antimalarial drugs such as primaquine, which could interfere with radical treatment. The present study reports for the first time the association of genetic factors and G6PD deficiency in Sri Lanka. Twelve single nucleotide polymorphisms (SNPs) were genotyped in 130 G6PD deficient individuals and 170 healthy controls. Association between the genotypes/alleles and G6PD deficiency was assessed. Unadjusted and adjusted analyses revealed two putative associations of rs915941 and rs915942 with G6PD deficiency status. An empirical power analysis provided evidence of virtually $100 \%$ power of detecting these associations if they were true. In both SNPs, the homozygotes referring to the minor allele showed a decrease in risk of being G6PD deficient. In particular, cases had 1.733 times odds of having the A allele of rs915941 when compared to controls ( $95 \% \mathrm{CI}=1.247-2.409$ ). In rs915942, the odds of the $\mathrm{G}$ allele are 1.625 times higher in cases when compared to controls (95\% CI $=1.199-2.202)$. Linkage disequilibrium revealed that these two SNPs are highly linked in these populations. Other studied SNPs were also in linkage and formed separate haplotype blocks. The detected associations of rs915941 and rs915942 with G6PD deficiency are likely to be true associations in these Sri Lankan study populations. Therefore, it is recommended to investigate the possible interaction of these SNPs in a primaquine treatment setting.
\end{abstract}

Keywords: Haemolysis, malaria, WST-8/1-methoxy PMS method.

\section{INTRODUCTION}

Sri Lanka was certified a malaria free nation by the World Health Organization in 2016 (WHO, 2016). Although the last indigenous malaria case was reported in 2012 by the Anti-Malaria Campaign (AMC, 2015) the threat has not ceased completely as imported cases continue to be reported (AMC, 2020). Despite the parasite surveillance and vector control programmes, there is every possibility of resurgence of the disease, which can progress into an epidemic. Therefore, timely detection and proper management of patients to ensure radical cure remains critically important for the country in order to remain as 'Malaria free'. Plasmodium vivax is considered more difficult to expel as they form 'hypnozoites' in liver cells which can stay dormant for months or even years before relapsing after the primary infection, acting as a source of transmission (Hulden \& Hulden, 2011; Adams $\&$ Mueller 2017). Primaquine is the only effective drug against hypnozoites and required for the elimination of gametocytes of $P$. falciparum infections. Thus, primaquine plays a critical role in achieving radical cure of the disease and an important factor concerning the treatment of imported malaria patients. However, the drawback of this drug is that it triggers haemolytic anaemia in individuals with glucose-6-phosphatedehydrogenase (G6PD) deficiency (Rehman et al., 2018). G6PD is an important enzyme present in all cells. It

\footnotetext{
* Corresponding author (rajika@parasit.cmb.ac.lk; (DD https://orcid.org/0000-0002-2041-6017)
} 
catalyses the first step in the hexose monophosphate pathway producing nicotinamide adenine dinucleotide phosphate (NADPH), which is important for many biosynthetic reactions. Deficiencies in G6PD enzyme give rise to various clinical manifestations including neonatal jaundice and acute haemolytic anaemia (Luzzatto et al., 2011). Certain drugs, i.e. primaquine and drugs containing sulfur, certain kinds of infections or foods can trigger haemolysis in G6PD deficient individuals (Luzzatto et al., 2011). G6PD deficiency is caused by point mutations in the G6PD gene. Therefore, such genetic mutations are of practical relevance to malaria-infected individuals since selected anti-malarials (primaquine) can trigger haemolytic anaemia in such individuals. Furthermore, mutations of this gene are also believed to be associated with susceptibility/resistance to malaria (Louicharoen et al., 2009; Dewasurendra et al., 2015).

There are more than 300 different enzyme variants based on diverse biochemical characteristics suggestive of variability in mutations in the G6PD gene, and over 180 genetic mutations reported worldwide (Peng et al., 2015). Most mutations are single point mutations, which lead to amino acid substitutions. The majority of mutations disrupt the structural stability of the enzyme and thus reduces its overall activity.

Existence of 17 single nucleotide polymorphisms (SNPs) in the G6PD gene in Sri Lankan study samples was first reported in 2015 (Dewasurendra et al., 2015). Gunawardena et al. in 2017 further described the prevalence of G6PD enzyme deficiency in two previously malaria endemic areas (Anuradhapura and Kurunegala) in Sri Lanka as $13.9 \%$ and $7.9 \%$, respectively (Gunawardena et al., 2017). The association between host genetic factors, i.e. G6PD gene polymorphisms and G6PD deficiency, has not been studied previously in Sri
Lankan study populations. The objective of this study was to explore the polymorphic SNPs found in selected Sri Lankan study samples and to identify any association between these SNPs and G6PD deficiency in the selected samples from these two districts.

\section{METHODOLOGY}

\section{Sample collection}

Sample populations for the first phase of this study were selected from individuals attending the Teaching Hospitals at Anuradhapura and Kurunegala between November 2013 and June 2014, as published in detail previously (Gunawardena et al., 2017). The recruited study sample $(n=2,059)$ was assessed for their G6PD enzyme status using the WST-8/1-methoxy PMS method. In this study sample, 142/1018 (13.95\%) and 83/1041 (7.97 \%) were G6PD deficient in Anuradhapura and Kurunegala Districts, respectively. Females were equally affected as males in each district: 35/313 $(11.18 \%)$ males and 107/705 (15.18\%) females were affected in Anuradhapura $(\mathrm{p}=0.089) ; 25 / 313(7.99 \%)$ males and 58/728 $(7.97 \%)$ females were affected in Kurunegala $(\mathrm{p}=0.991)$. The 45 individuals (28 from Anuradhapura District and 17 from Kurunegala District) with severe enzyme deficiency (enzyme activity $<10 \%$ ) were included in the selected sample for genotyping. The remaining cases $(n=85)$ were those selected from among individuals who were moderately deficient for G6PD enzyme activity (enzyme activity $10-30 \%$ ) in the initial phase $(n=180)$. The total number of cases and controls selected for genotyping were 130 and 170 , respectively (Table 1).

Ethical clearance was obtained from the Ethics Review Committee (ERC), Faculty of Medicine, University of Colombo (EC-12-13/EC-14-011) and

Table 1: Number of cases and controls from each district selected for genotyping (number successfully genotyped / number selected for genotyping).

\begin{tabular}{lccccc}
\hline District & Gender & Cases & Controls & Total & $\begin{array}{c}\text { Total for } \\
\text { each district }\end{array}$ \\
\hline Anuradhapura & Male & $14 / 16$ & $17 / 24$ & $31 / 40$ & \\
& Female & $42 / 44$ & $54 / 56$ & $96 / 100$ & $127 / 140$ \\
Kurunegala & Male & $24 / 24$ & $36 / 36$ & $60 / 60$ & \\
& Female & $43 / 46$ & $52 / 54$ & $95 / 100$ & $155 / 160$ \\
& Total & $123 / 130$ & $159 / 170$ & $282 / 300$ & \\
\hline
\end{tabular}


the ERC of Teaching Hospital Kurunegala. Permission for sample collection was obtained from the relevant administrative bodies of the Ministry of Health and the respective hospitals. Written informed consent was obtained from all the participants and proxy consent was obtained from the parents/guardians of subjects aged less than 18 years. Each individual was given a unique identification number. Two drops of blood $(\sim 0.5 \mathrm{~mL})$ from each individual were collected on to $3 \mathrm{MM}$ filter papers (Whatman) and air dried before storing individually in zip-lock bags containing silica gel. These were stored at $4{ }^{\circ} \mathrm{C}$ until transported (within 7 days in ice boxes) to the Department of Parasitology, Faculty of Medicine, University of Colombo. Dried blood spots stored at $4{ }^{\circ} \mathrm{C}$ can retain its reactive intensity and enzyme activity up to 10-14 days (Tantular et al., 2003; Kuwahata et al., 2010; Niz et al., 2013). Data on age and gender of each individual were also collected.

\section{G6PD functional assay}

G6PD enzyme activity detection assay, which was based on the previously validated modified WST-8/1-methoxy PMS method described by Kuwahata et al. (2010) was carried out as described elsewhere (Gunawardena et al., 2017). This method was modified by Kuwahata et al. (2010) to be used in a 96-well plate format with dried blood spots and validated for mass screening of G6PD functional deficiency (Tantular et al., 2003; Kuwahata et al., 2010). Evaluation of the qualitative performance of the WST-8/1-methoxy PMS method against spectrophotometry (Trinity Biotech, Ireland) has shown sensitivity and specificity rates of $88.9 \%$ and $95.6 \%$ at the $10 \%$ enzyme activity level and $84.2 \%$ and $98.2 \%$ at the $30 \%$ level, respectively (Espino et al., 2016). In brief, the WST-8/1-methoxy PMS mixture was purchased from Dojindo Laboratories (Japan), while the commercial standard G6PD normal control (Trinity Biotech, Ireland) was used to create the panel of internal controls for each assay (normal, moderately deficient: $\sim 30 \%$ enzyme activity, severely deficient: $\sim 10 \%$ enzyme activity). Plates were incubated for $2 \mathrm{~h}$ at ambient temperature $\left(\sim 30{ }^{\circ} \mathrm{C}\right)$, and were then inspected visually by two separate observers. For quantitative analysis, the optical density was quantified in a micro-plate reader at OD 450-630 nm. The method was further validated on 20 samples sent to a commercial laboratory (Colombo, Sri Lanka) that used a kinetic UV determination test kit for determining G6PD status (Biochemical Enterprise). One hundred and thirty individuals who were with either severe ( $\mathrm{n}=45 ;<10 \%$ enzyme activity) or moderately deficient ( $\mathrm{n}=85 ; 10-30 \%$ enzyme activity) G6PD enzyme activity were selected as cases for genotyping. Another 170 individuals from the same areas with normal enzyme activity were selected as controls.

\section{DNA extraction and genotyping}

DNA extraction of the samples $(\mathrm{n}=300$; i.e. 130 cases - 40 males and 90 females; 170 controls - 60 males and 110 females) was carried out using the dried blood spots with commercial DNA extraction kits (QIAmp blood

Table 2: The 12 SNPs selected for genotyping and their chromosomal positions (according to the Ensemble genome browser, release 104 May 2021). The minor/ major alleles with the minor allele frequencies are also indicated

\begin{tabular}{clcc}
\hline & SNP rs number & Chromosomal position & $\begin{array}{c}\text { Minor/Major alleles } \\
\text { (minor allele freq.) }\end{array}$ \\
\hline 1 & rs766420 & 154326058 & $\mathrm{G} / \mathrm{C}(0.462)$ \\
2 & rs915941 & 154398308 & $\mathrm{C} / \mathrm{A}(0.097)$ \\
3 & rs915942 & 154398397 & $\mathrm{~A} / \mathrm{G}(0.092)$ \\
4 & rs2071429 & 154532293 & $\mathrm{G} / \mathrm{A}(0.443)$ \\
5 & rs2230037 & 154532439 & $\mathrm{~A} / \mathrm{G}(0.401)$ \\
6 & rs4898389 & 154599376 & $\mathrm{G} / \mathrm{A}(0.447)$ \\
7 & rs5986877 & 154600008 & $\mathrm{G} / \mathrm{C}(0.437)$ \\
8 & rs7879049 & 154601444 & $\mathrm{G} / \mathrm{A}(0.382)$ \\
9 & rs7053878 & 154605847 & $\mathrm{~A} / \mathrm{T}(0.114)$ \\
10 & rs72554665 (G6PD Canton) & 154532269 & Non polymorphic \\
11 & rs5030868 (G6PD Mediterranean) & 154534419 & Non polymorphic \\
12 & rs137852314 (G6PD Mahidol) & 154534495 & Non polymorphic \\
\hline
\end{tabular}


micro kit-cat\# 56304, QIAGEN, Germany) as instructed by the manufacturer. DNA samples were sent to LGC genomics Ltd., UK for commercial genotyping using the Kompetitive Allele Specific PCR system (KASP ${ }^{\mathrm{TM}}$ ) (LGC Limited, n.d.; Ertiro et al., 2015). The samples were genotyped to identify 12 polymorphic SNPs (Table 2) which have previously been documented in Sri Lankan study populations (Dewasurendra et al., 2015).

\section{Data analysis and power calculations}

Data were analysed using the statistical software $R$ (V3.5.0, R Foundation for Statistical Computing, Vienna, Austria), SPSS (IBM SPSS Statistics for Windows, Version 19.0. Armonk, NY: IBM Corp), GenePop V 4.2 (Laboratiore de Genetiqueet Environment, Montpellier, France) and Haploview V 4.2. (Broad Institute, Boston, MA, USA).

Out of 300 individuals, only 282 (i.e. 123 cases - 38 males and 85 females; 159 controls - 53 males and 106 females) were selected for further analysis as genotyping was not successful in 18 subjects. Three commonly known SNPs associated with G6PD deficiency (i.e. rs72554665 - G6PD Canton, rs5030868 - G6PD Mediterranean and rs137852314 - G6PD Mahidol) were monomorphic for this study sample and were excluded from further analysis.

Since the G6PD gene is located in the $\mathrm{X}$ chromosome, the initial analysis was done separately for males and females. For each gender, Pearson's chi-square tests for independence in two-way tables were firstly performed to test the association between alleles/genotypes and the G6PD enzyme status (G6PD deficient-cases, G6PD activity normal-controls). An adjusted analysis was then performed in order to control for potential confounding effects. In this analysis, a non-genetic logistic regression model describing the log odds of being G6PD enzyme deficient as a function of gender, age and location was fitted to the data. For each SNP, a genetic logistic regression model was then fitted to the same data. This model included the above covariates and a general effect of each genotype associated with the SNP under analysis. To formally test the genetic association between a specific SNP and the outcome, the non-genetic and genetic models were compared against each other using log-likelihood ratio tests. In this test, high values of $-\log _{10}(\mathrm{p}$ value) were indicative of a putative association between a given SNP and the G6PD enzyme status. To control for multiple testing in the adjusted analysis, the Benjamini-Hochberg procedure was applied to all the $p$ values generated from each SNP association analysis so a global false discovery rate of $5 \%$ could be achieved for the analysis. This procedure was based on the following algorithm: (i) order the $p$ values from the lowest to the highest, (ii) calculate the largest $\mathrm{k}$ that satisfies $\mathrm{p}_{\mathrm{k}}<0.05 / \mathrm{k}$ where $\mathrm{p}_{\mathrm{k}}$ is the $\mathrm{k}^{\text {th }}$ ordered $\mathrm{p}$ value; (ii) reject the null hypothesis for all ordered $\mathrm{p}$ values lower than $\mathrm{p}_{\mathrm{k}}$. Controlling for multiple testing was not conducted in the unadjusted analysis because the final results were solely based on the adjusted one. Additive, dominant and recessive effects could have been accounted for in this analysis. However, these genetic effects are typically more adequate to analyse autosomal SNPs or to analyse data from females only. Analysing females separately $(\mathrm{n}=200)$ was not done because it would lead to a reduction of statistical power to detect a given genetic association.

For the better understanding of the power of this study in detecting putative genetic associations, an empirical power analysis was carried out using data simulation. With this purpose, the fitted genetic model associated with each SNP was assumed to be the true one for the data. This model was then used to simulate a new phenotype for each individual in the sample by randomly generating a Bernoulli trial with success probability given by the respective estimated probabilities of being G6PD enzyme deficient. A thousand new datasets were generated in this way while maintaining all covariates fixed at their original values. In each of the simulated datasets, the association analysis was repeated as described above. In the end, the power to detect an association between a given SNP and the phenotype was estimated by the percentage of tests that preferred the genetic model (including non-genetic covariates and genetic effects) when compared to the non-genetic model (including non-genetic covariates only).

Linkage disequilibrium and haplotypes were determined for the whole study sample as well as for the sample groups from each district and between sexes using Haploview V 4.2.

\section{RESULTS AND DISCUSSION}

This was the second phase of the study conducted to determine the extent of G6PD deficiency in a Sri Lankan study population (Gunawardena et al., 2017). Analysis of demographic data and their association with G6PD deficiency status of this study population has been published earlier (Gunawardena et al., 2017). The subsample which was extracted from this initial study 
population $(n=300)$ consisted of 200 females (100 from each district) and 100 males (40 from Anuradhapura and 60 from Kurunegala). In females, the ages ranged from 3-83 years (mean 52.23 years); while in males, ages ranged from $2-79$ years (mean 52.13 years).

Individuals identified as functionally deficient in G6PD enzyme activity $(<30 \%)$ as well as controls with normal enzyme activity (> $60 \%$ ) were selected for this study. A previous study that genotyped 68 SNPs in the G6PD gene, which included variants well known to cause G6PD deficiency, found most of them to be monomorphic in the Sri Lankan study population (Dewasurendra et al., 2015). Out of the 68 SNPs, 9 that were polymorphic and with MAF $>0.1$ were selected for genotyping in this study together with 3 other SNPs known to occur in Asia/South Asia (Canton, Mahidol and Mediterranean).

\section{Association of genotypes and alleles with G6PD enzyme deficiency status in gender stratified sub samples}

Gender stratified analysis was carried out for the whole study sample as well as for sub samples from each district. There was no evidence of genotypic deviation from the Hardy-Weinberg equilibrium ( $p>0.05)$.

\section{For the male study population}

The allele distribution of rs915941 was significantly different among cases and controls in males of the entire study sample. All cases possessed allele A (alternative allele/ mutation; the major allele) of rs915941 in contrast to only $89.8 \%$ of controls $\left(\chi^{2}=4.009, p=0.0453\right)$.

This SNP is in a $5^{\prime}$ untranslated region (5' UTR variant), which is important for translation of a transcript. A mutation $(\mathrm{C}>\mathrm{A})$ in this SNP is capable of causing alterations in the final product of the protein synthesis pathway.

Similarly, for rs915942, the percentage of male individuals with the ancestral allele $(\mathrm{G})$ in cases was significantly higher when compared to the controls, thus hinting at a 'protective effect' of this mutation $\left(\chi^{2}=\right.$ $4.009, \mathrm{p}=0.0453$ ). All cases possessed allele $\mathrm{G}$ (ancestral allele; the major allele) of rs 915942 while only $89.8 \%$ of controls had the ancestral $\mathrm{G}$ allele.

This is a splice region variant $(\mathrm{G}>\mathrm{A})$, indicative of a genetic mutation that may insert/delete/change nucleotides during the splicing of precursor mRNA into mature mRNA. A mutation in this region may lead to abnormalities of proteins that could lead to enzyme deficient status.

The results of the logistic regression model applied to the whole study sample further substantiates the above observations on rs915941 and rs915942. However, this significance for these 2 SNPS could not be observed when the male study group was further divided into sub groups from each district (viz. Anuradhapura, Kurunegala districts) probably due to the limitation in numbers.

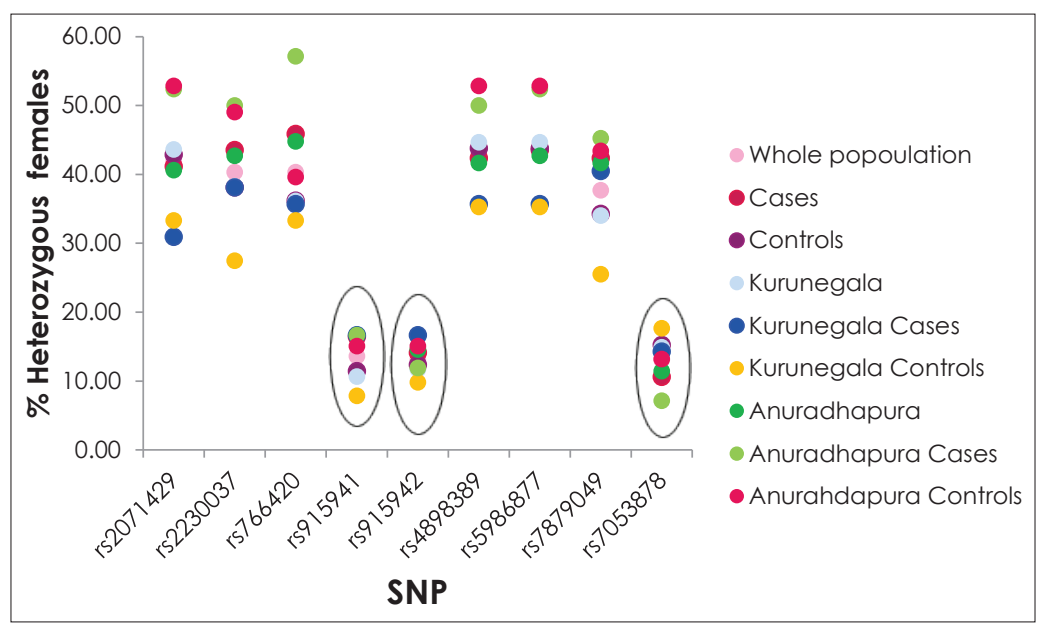

Figure 1: The percentage heterozygous females for each SNP. The low percentages of heterozygous females for rs915941, rs915942 and rs7053878 are encircled. 


\section{For the female study population}

There were both heterozygous and homozygous females in deficient and non-deficient groups, but no significant differences in percentages of these heterozygous/ homozygous individuals could be observed between the two groups as a whole or within each district. However, the percentages of heterozygous females for rs 7053878 , rs915941 and rs915942 were low compared to the percentages of heterozygous females for the other 7 SNPs (Figure 1). Heterozygous females were defined as individuals with a genotype consisting of two different alleles and homozygous were defined as individuals with two identical alleles at a particular locus.

Generally, heterozygous females would be the most at advantage with protection offered against mal-effects of G6PD deficiency as well as from morbidity and mortality due to severe malaria (Manjurano et al., 2015).

Thus, a higher proportion of heterozygous females would have been expected in this study sample. However, contrary to this assumption, the heterozygous proportion of females depicted in the three SNPs, which hint at an association with G6PD deficiency was very low compared to the other 9 SNPs studied. There are studies that indicate G6PD enzyme deficiency to be protective for hemizygous males but not heterozygous females against severe malaria (Guindo et al., 2007), while homozygous females have shown to be protected from severe malaria (Greene, 1993). Homozygous females act similar to hemizygous males for G6PD deficiency due to gene mosaicism in $\mathrm{X}$ chromosome gene variants. Since this study sample was selected from two districts that had withstood a high malaria burden over the centuries, there is every possibility of 'positive selection' of homozygous females over heterozygous females as seen in this study sample.

Minor allele frequencies (MAF) varied from 0.00 to 0.49 , with the highest reporting for rs5986877 in female cases from Kurunegala. The MAF of rs 2071429 was higher $(\mathrm{MAF}>0.4)$ in both female cases and female controls in both districts when compared to MAF of other SNPs, and relatively lower MAF could be observed for rs7053878, rs915941 and rs915942 in all groups (Figure 2).

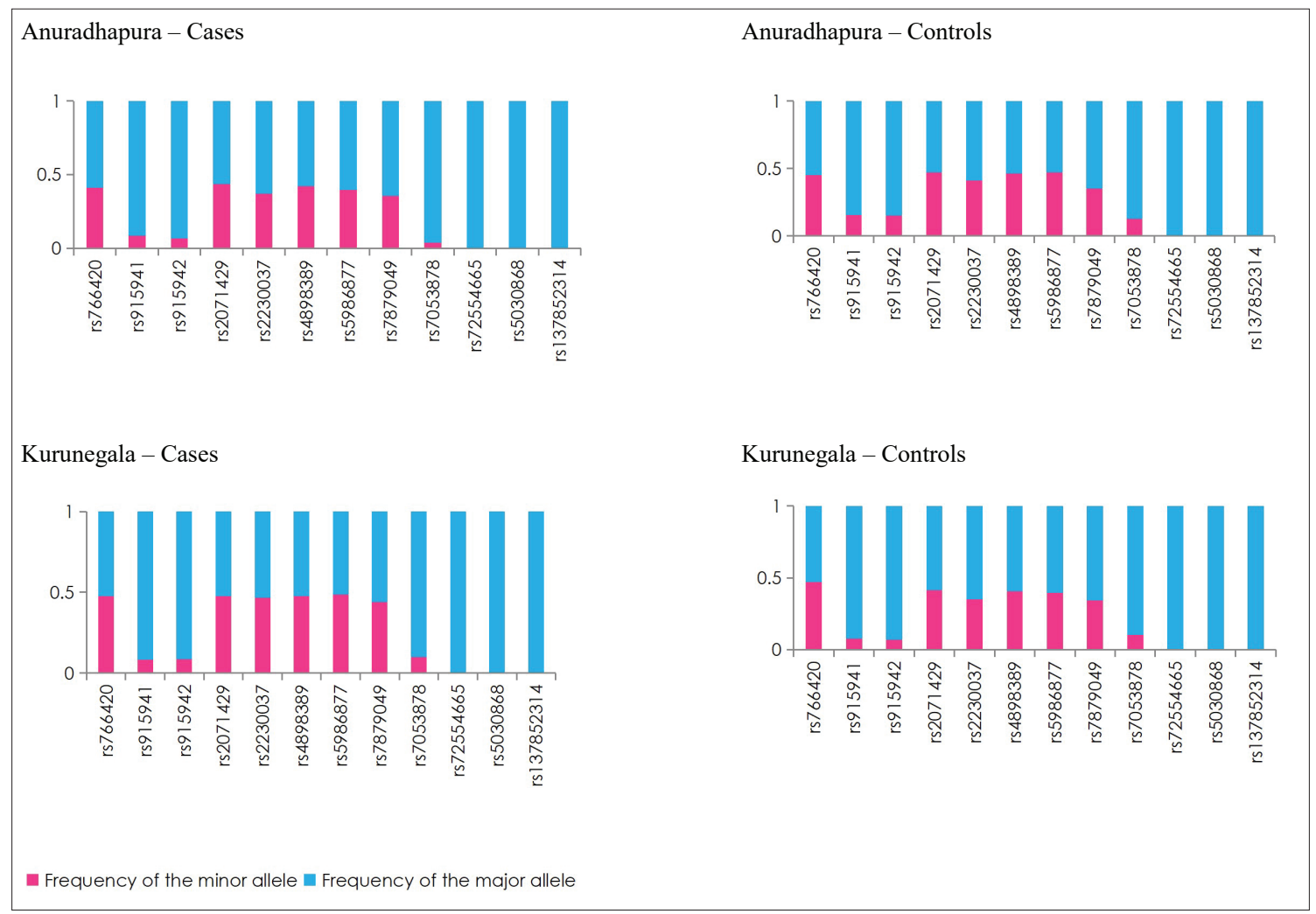

Figure 2: Minor allele frequencies of the 12 SNPs studied in female cases and female controls in the 2 districts. The pink stack bar indicates the minor allele frequency. 
Analysis for associations of genotypes/alleles with enzyme deficiency considering the whole female study group showed no significant difference between cases and controls. However, in females of Anuradhapura, the frequency of the $\mathrm{T}$ allele (ancestral/wild type allele) of the marker rs7053878 was significantly greater in the deficient group $(96.1 \%)$ in contrast to the normal group $(87.3 \%) ;(\chi 2=4.12, \mathrm{df}=2, \mathrm{p}=0.042)$. This was not observed among females in Kurunegala. This SNP (rs7053878) is an upstream gene variant, which is classified as a 'modifier', which usually is a non-coding variant or a variant affecting a non-coding gene. The mutation could be either $\mathrm{T}>\mathrm{C}$ or $\mathrm{T}>\mathrm{A}$ depending on the population; this study population possessed the $\mathrm{T}>\mathrm{A}$ mutation.

\section{Genetic association analysis adjusted for possible confounding factors}

When all the study subjects were considered as a single study group (both males and females together) the presence of alleles/genotypes of two markers, i.e. rs915941 and rs915942 were significantly different $(p<0.01)$ between cases and controls (Figure 3A;

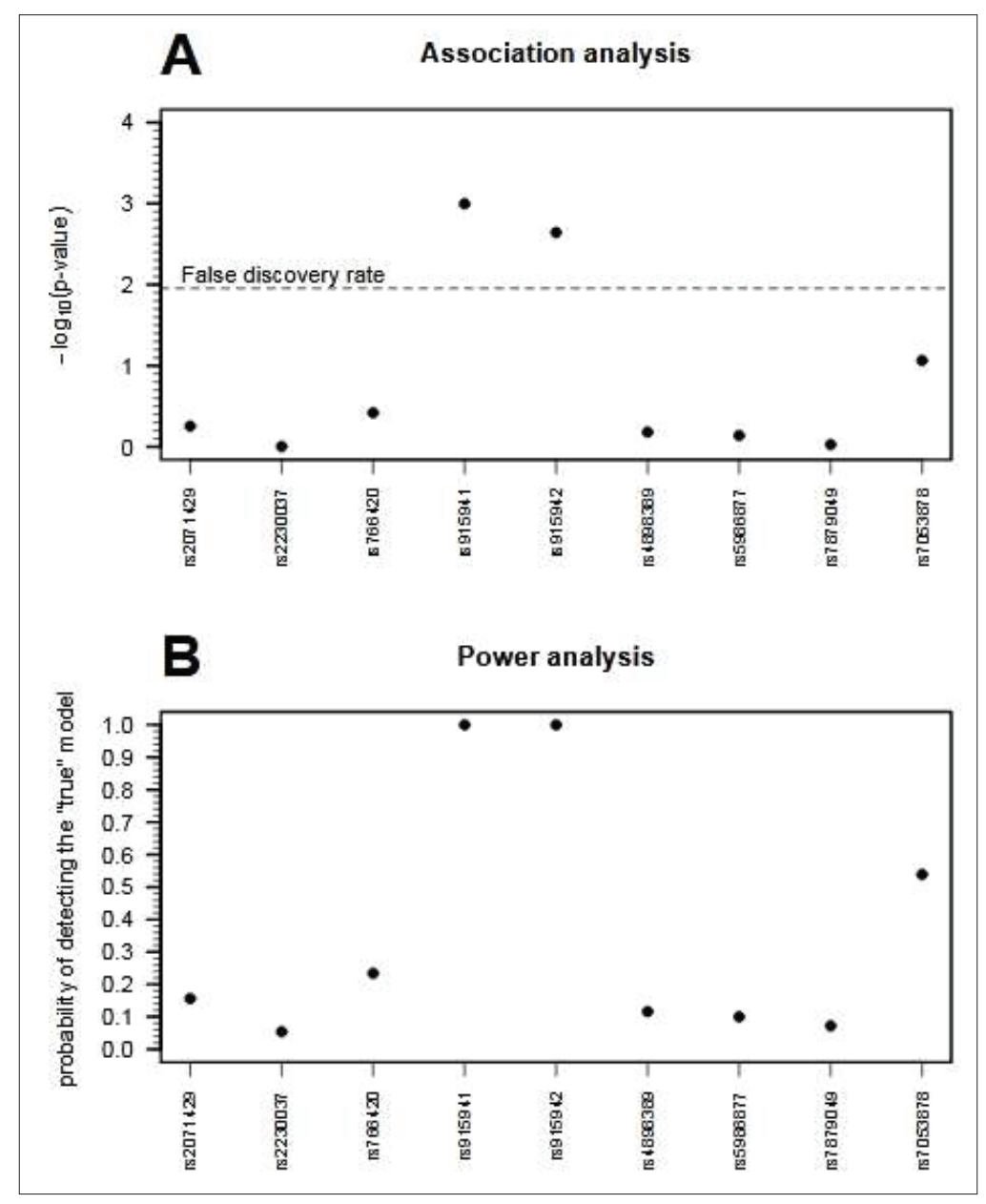

Figure 3: Association analysis between different G6PD SNP and G6PD enzyme status. A: Association signals measure as $-\log _{10}$ ( $p$ value) with respect to the likelihood ratio test comparing non-genetic and genetic models for the data. The horizontal dashed line represents the threshold for statistical significance based on a global $5 \%$ false discovery rate. B: Power calculations to detect an association between each SNP and G6PD enzyme deficient status under the assumption that the true model for each SNP was the one associated with the analysis shown in A. 
Table 3), while neither gender nor district had any significant effect upon the enzyme activity $(p>0.05)$. Relative risk of an individual having the A allele of rs915941 in cases was 1.733 times higher compared to that of controls ( $95 \% \mathrm{CI}=1.247-2.409$ ). Similarly, in rs915942 the relative risk of an individual having the $G$ allele in cases was 1.625 times higher compared to that of controls (95\% CI $=1.199-2.202)$. The proportion of total genetic variance was similar between the two groups, cases and controls $\left(\mathrm{F}_{\mathrm{ST}}=0.000351\right)$. For both SNPs, the homozygotes for the minor allele showed a reduced risk of being G6DP enzyme deficient (Table 3). However, the standard errors of the respective genetic effects produced unreliable estimates because the homozygotes of the minor allele were all G6PD enzyme normal. Similar statistical phenomenon is frequently observed when analysing contingency tables in the presence of zeros.

Table 3: Coefficient estimates of the genetic models associated with rs915941 and rs 915942 where A and B represent the wild type and alternative alleles (rs915941-A/C; rs915942-G/A), respectively, $\mathrm{SE}$ is the standard error of the respective estimate.

\begin{tabular}{lcccc}
\hline Coefficient & \multicolumn{2}{c}{ rs915941 } & \multicolumn{2}{c}{ rs915942 } \\
& Estimate (SE) & p value & Estimate (SE) & p value \\
\hline Intercept & $-1.45(0.45)$ & $<0.01$ & $-1.40(0.45)$ & $<0.01$ \\
Age (in years) & $0.03(0.01)$ & $<0.01$ & $0.02(0.01)$ & $<0.01$ \\
Gender (male) & $-0.01(0.28)$ & 0.98 & $0.03(0.28)$ & 0.92 \\
District (Kurunegala) & $-0.16(0.26)$ & 0.54 & $-0.11(0.26)$ & 0.66 \\
SNP & & & & \\
\multicolumn{1}{c}{ AB } & $0.14(0.41)$ & 0.73 & $-0.02(0.42)$ & 0.96 \\
\multicolumn{1}{c}{ BB } & $-16.41(697.21)$ & 0.98 & $-16.40(732.82)$ & 0.98 \\
\hline
\end{tabular}

Since lack of association between a given SNP and the phenotype could be due to a small sample size, a power calculation was conducted for each SNP under the assumption that the respective fitted genetic model was the true one for the data. The respective results revealed that the sample size used in this study was only able to detect the true model with a probability up to $50 \%$ for the SNPs not detected in the adjusted association analysis (Figure 3B). For rs915941 and rs915942, the power to detect the respective associations was close to $100 \%$.

In both SNPs, rs915942 (for male study sample) and rs7053878 (for female study sample from Anuradhapura District), the percentage of individuals with the ancestral allele was significantly higher in cases (for rs915942; $p=0.0453$ and for rs 7053878; $p=0.042$ ) when compared to controls indicating a possible association of the ancestral allele with G6PD deficiency and the protective nature of the alternate allele against deficiency. This queries the fact as to whether these SNPs have a direct effect on enzyme deficiency or whether they appeared linked to the disease through linkage with other SNPs. In this study sample, rs915942 was highly linked with rs915941 (Figures 4A and 4B), but rs7053878 was not linked to any of the tested SNPs. However, Maiga et al. (2014) and Manjurano et al. (2015) reported rs7053878 being highly linked to rs1050828 (G6PD202) and rs1050829 (G6PD376), which are well known genetic variants that cause G6PD deficiency in African study populations. This highlights the possibility of rs 7053878 acting together with other SNPs in the G6PD gene to generate the final outcome of the effect of these SNPs on G6PD deficiency. Both these variants (G6PD202 and G6PD376) were genotyped earlier in a different study population $(\mathrm{n}=1,051)$ and found to be monomorphic (Dewasurendra et al., 2015) and thus, not included in the current association study. However, the possibility exists that a minor proportion of this study population maybe polymorphic for these two known variants which could be responsible for the G6PD deficiency in this study population, and the linkage of rs7053878 with these variants could generate the signal that was seen in this analysis.

In analysing the data, additive, dominant and recessive effects could have been accounted for in this analysis but as described earlier, these genetic effects are more appropriate for analysing autosomal SNPs or for 
analysing genetic data of female study populations only. However, the analysis of a relatively smaller female study sample $(n=200)$ could lead to a reduction of statistical power to detect a given genetic association.

\section{Linkage disequilibrium and haplotypes}

Simultaneous evidence of an association and lack of it for different subsets of SNPs in the same locus might be attributed to different haplotype structures and/or linkage disequilibrium between different SNPs. When the whole study sample was considered, a strong linkage could be observed between the two SNPs rs915941 and rs915942 ( $\left.\mathrm{D}=0.973, \mathrm{r}^{2}=0.946\right)$ as well as five other SNPs, i.e. rs2071429, rs2230037, rs4898389, rs5986877 and rs7879049 (all pairs with D 1.0) which formed a separate haplotype block.
Differences could be observed both in linkage disequilibrium (LD) as well as in haplotypes of the tested markers between cases and controls (Figures 4A and 4B). Among individuals with normal enzyme activity, a strong linkage $\left(\mathrm{D}^{\prime}=1.0, \mathrm{r}^{2} \geq 0.97\right)$ could be observed between 5 of the tested SNPs, i.e. rs2071429, rs2230037, rs4898389, rs5986877 and rs7879049, which formed a single haplotype block. The SNP rs766420 was in high LD with rs915942 ( $\mathrm{D}^{\prime}=1.0, \mathrm{r}=0.96$ ). Among cases, however, a strong LD could be observed between rs915941 and rs915942, which formed a second block in addition to the first haplotype block seen in controls. Recombination rate of the two blocks was $44 \%$. Furthermore, linkage between rs766420 and rs915942 was poor in G6PD deficient individuals ( $\mathrm{D}^{\prime}=0.61$, $\left.r^{2}=0.038\right)$ when compared to controls ( $D^{\prime}=0.918$, $\left.\mathrm{r}^{2}=0.121\right)$.

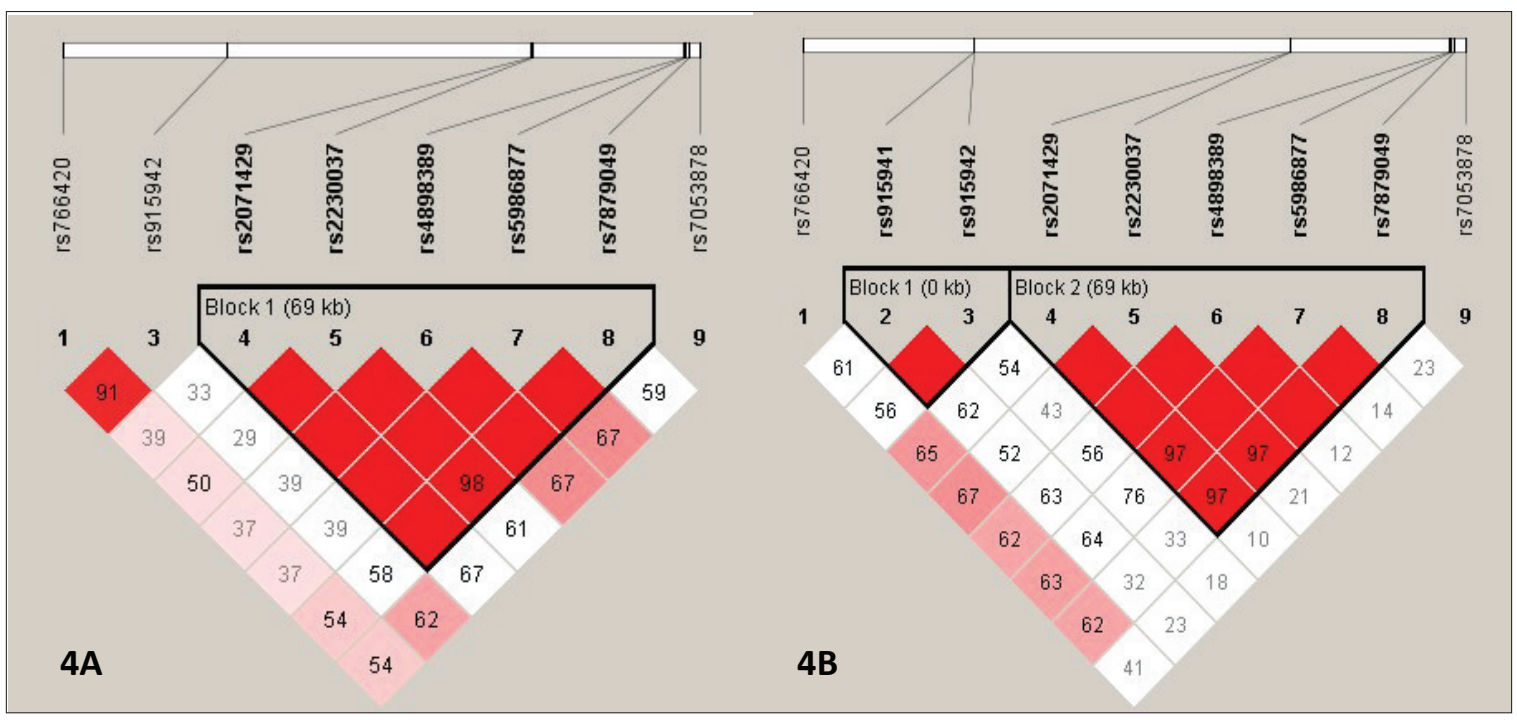

Figure 4: Linkage disequilibrium plots for, A) controls and B) cases. The red squares indicate high linkage $(\mathrm{D}>0.9)$ and pink squares indicate SNPs in moderate linkage. SNPs in low/no linkage are indicated by white squares

Linkage between rs915941, rs915942, rs7053878 (which seemed to be significantly associated with G6PD enzyme activity) and any other marker present in the G6PD gene was checked for in the Sri Lankan population (using a dataset from a previous study) as well as for other populations (using Ensemble genome browser). Both rs915941 and rs915942 were in moderate LD (LOD 70) with rs5986877, while rs7053878 was in moderate LD with many SNPs including rs7879049, rs5986877, rs4898389, rs2230037 and rs2071429 in a previously analysed Sri Lankan study sample (Dewasurendra et al., 2015). While moderate linkage was identified between rs915942 and rs7053878 $\left(\mathrm{r}^{2}=0.451\right)$, the LD between rs915942 and other polymorphic markers was weak $\left(r^{2}<0.04\right)$. Evidence was scarce for the occurrence of linkage between any of the other known markers present in the G6PD gene. 
Linkage of these 3 SNPs (i.e. rs915941, rs915942 and rs 7053878) with other markers present in the G6PD gene was tested in Sri Lankan study populations (using previous data published in Dewasurendra et al., 2015) as well as for other study populations, i.e. Kenyan, South Asian, East Asian and American (Zerbino et al., 2018). SNPs rs915941, rs915942 and rs7053878 were found to be in linkage with some of the SNPs in the G6PD gene in Sri Lankan study populations. But none of these SNPs are known to be associated with G6PD deficiency. At the global level, these 3 SNPs were found to be in high linkage with rs2070827 in Kenyan study populations, and with many other SNPs (n > 40) in South and East Asian study populations as well as American study populations (Zerbino et al., 2018). Nonetheless, none of these markers in linkage were associated with G6PD deficiency (except the linkage of rs 7053878 with G6PD202 and G6PD376 variants described above).

Considering study samples from each district, genetic markers in male/female groups of Anuradhapura showed some association with G6PD deficiency. This was not evident in Kurunegala. Gunawardena et al. revealed that the prevalence of G6PD deficiency was much higher ( 2 times) in Anuradhapura when compared to Kurunegala (Gunawardena et al., 2017). Thus, there could be genetic and non-genetic factors that might be involved in determining the prevalence of enzyme deficiency in this study group. It could be hypothesised that the SNP rs915941 might be associated with the higher prevalence of enzyme deficiency that was apparent in Anuradhapura.

Haldane's malaria hypothesis proposing malaria as an evolutionary driver for the distribution of certain genetic variants appears to hold true for G6PD deficiency (Haldane, 1949; Hedrick, 2011). Protection against severe malaria is offered by the African variant G6PD A-(Liang et al., 2019) while the frequencies of the Mediterranean and Asian variants are greater in areas where $P$. vivax is endemic (Louicharoen et al., 2009; Dewasurendra et al., 2015). The association of several G6PD gene variants with malaria in a Sri Lankan study population was also reported recently (Dewasurendra et al., 2015). Malaria has been endemic from historical times in the Dry Zone of Sri Lanka with epidemics occurring at periodic intervals (Fernando, 2014; Silva, 2014) although indigenous malaria transmission is no longer present (WHO, 2016). Anuradhapura (North-Central Province) and Kurunegala (North-Western Province) are two such districts situated in the Dry Zone of Sri Lanka which have recorded high malaria endemicity in the past (AMC, 2015). Thus, the G6PD deficiency condition despite being detrimental, could have offered a selective survival advantage over normal enzyme levels against morbidity and mortality of malaria (Manjurano et al., 2015). This may explain the significantly higher presence of certain ancestral SNPs in cases compared to controls.

Furthermore, the phenomenon of gene mosaicism in females could have had an effect upon the analysis of our study. Random inactivation of one of the two inherited $\mathrm{X}$ chromosomes occurs in females (gene mosaicism). Therefore, although genotyped as heterozygous the actual proportion of females that express the gene activity maybe variable. As such, the relationship between the marker and the disease status may not be so evident.

Results of the first phase of this study which determined the prevalence of G6PD deficiency in Sri Lanka (Gunawardena et al., 2017) provides strong evidence for the occurrence of phenotypic disease in both males and females, especially in Anuradhapura; while the current research indicates the possibility of host genetic markers that may be associated with G6PD deficiency in both male and female groups. Since antimalarials can cause haemolysis in G6PD deficient individuals, screening for G6PD enzyme status in both males and females is important prior to initiating treatment, especially in previously high malaria endemic areas in order to prevent re-introduction of malaria to Sri Lanka.

\section{CONCLUSION}

The G6PD deficiency status in Sri Lankan populations might be influenced by genetic factors. The single nucleotide polymorphisms in the G6PD genes rs 7053878, rs915941 and rs915942 appear to be associated with G6PD enzyme activity. Individuals with the ancestral allele of rs7053878 (in females) and rs915942 (in males) were relatively more likely to have G6PD enzyme deficiency when compared to individuals with the alternate allele, while individuals with the alternate allele of rs915941 (in males) were more likely to have the disease compared to those individuals with the ancestral allele. We conclude that the detected associations of rs915941 and rs915942 with G6PD deficiency are likely to be true associations in these Sri Lankan study populations.

Screening for G6PD enzyme deficiency in both males and females is important before administration of antimalarials or other specific drugs which could lead to haemolysis. 
Furthermore, it is recommended to investigate the possible interactions of these SNPs in a primaquine treatment setting.

\section{Acknowledgements}

The authors acknowledge technical support provided by Mr G.M.G. Kapilananda, Ms Dilhani Samarakoon and Mr Sashika Maddevithana of the Department of Parasitology, Faculty of Medicine, University of Colombo. The authors thank Director General of Health Services and the Directors, Consultant Haematologists and the nursing staff of Teaching Hospitals Anuradhapura and Kurunegala for their support during this study. Anna Jeffreys of Wellcome Trust Centre for Human Genetics, University of Oxford, UK is acknowledged for the support given for transferring of the genetic material for genotyping. Funding provided by the National Research Council of Sri Lanka (Grant No: 14 - 069) is gratefully acknowledged.

\section{REFERENCES}

Adams J.H. \& Mueller I. (2017). The Biology of Plasmodium vivax. Cold Spring Harbor Perspectives in Medicine 7(9): a025585.

DOI: http://dx.doi.org/10.1101/cshperspect.a025585

Anti Malaria Campaign Sri Lanka (AMC) (2015). Annual Report 2015. Anti Malaria Campaign, Ministry of Health, Sri Lanka.

Anti Malaria Campaign Sri Lanka (AMC) (2020). Anti Malaria Campaign, Ministry of Health, Sri Lanka. Available at http://www.malariacampaign.gov.lk

Beutler E., Vulliamy T. (2002). Hematologically important mutations: glucose-6-phosphate dehydrogenase. Blood Cells, Molecules and Diseases 28(2): 93-103.

DOI: http://dx.doi.org/10.1006/bcmd.2002.0490

Biochemical Enterprise BEN S.r.1 . Available at https://www. bensrl.it/index.php

Dewasurendra R.L., Rockett K., Fernando S.D., Carter R., Kwiatkowski D.P., Karunaweera N.D. (2015). G6PD gene variants and its association with malaria in a Sri Lankan population. Malaria Journal 14: 93(2015). DOI: https://doi.org/10.1186/s12936-015-0603-9

Ertiro B.T., Ogugo V., Worku M., Das B., Olsen M., Labuschagne M. \& Semagn K. (2015). Comparison of Kompetitive Allele Specific PCR (KASP) and genotyping by sequencing (GBS) for quality control analysis in maize. BMC Genomics 16: 908(2015).

DOI: http://dx.doi.org/10.1186/s12864-015-2180-2

Espino F.E., Bibit J.A., Sornillo J.B., Tan A., von Seidlein L. \& Ley B. (2016). Comparison of three screening test kits for G6PD enzyme deficiency: Implications for its use in the radical cure of vivax malaria in remote and resource-poor areas in the Philippines. PLoS One 11(2): 1-12.
DOI: https://doi.org/10.1371/journal.pone.0148172

Fernando P. (2014). Past malaria epidemics in Sri Lanka - an analysis. Journal of College of Community Physicians Sri Lanka 19(1): 27-41.

Greene L.S. (1993). G6PD deficiency as protection against falciparum malaria: An epidemiologic critique of population and experimental studies. American Journal of Physical Anthropology 36(17 S): 153-178.

DOI: https://doi.org/10.1002/ajpa.1330360609

Guindo A., Fairhurst R.M., Doumbo O.K., Wellems T.E., Diallo D.A. (2007). X-linked G6PD deficiency protects hemizygous males but not heterozygous females against severe malaria. PLOS Medicine 4(3): e66. DOI: http://dx.doi.org/10.1371/journal.pmed.0040066

Gunawardena S., Kapilananda G., Samarakoon D., Maddewithana S., Wijesundera S., Goonaratne L.V. \& Karunaweera N.D. (2017). Prevalence of G6PD deficinecy in selected populations from two previously high malaria endemic areas of Sri Lanka. PLoS One 12(2): e0171208. DOI: https://doi.org/10.1371/journal.pone.0171208

Haldane J.B.S. (1949). The rate of mutation of human genes. Hereditas 35(S1): 267-273.

Hedrick P. (2011). Genetics of Populations. Jones \& Bartett Learning, USA.

Hulden L. \& Hulden L. (2011). Activation of the hypnozoites: a part of Plasmodium vivax life cycle survival. Malar Journal 10: 90(2011). DOI: https://doi.org/10.1186/1475-2875-10-90

Kuwahata M., Wijesinghe R., Ho M., Ho Mei-Fong, Pelecanos A., Bobogare A., Landry L., Bugora H., Valley A., McCarthy J. (2010). Population screening for glucose-6phosphate dehydrogenase deficientcies in Isabel Province, Solomon Islands, usng a modified enzyme assay on filter paper dried bloodspots. Malar Journal 9: 223(2010). DOI: http://dx.doi.org/10.1186/1475-2875-9-223

LGC Limited (n.d.). KASP Genotyping Chemisty User Guide and Manual. Available at http://www.lgcgroup.com/ LGCGroup/media/PDFs/Products/Genotyping/KASPgenotyping-chemistry-User-guide.pdf?ext=.pdf

Liang X.Y. et al. (15 authors) (2020). Evidence of positively selected G6PD A- allele reduces risk of Plasmodium falciparum infection in African population on Bioko Island. Molecular Genetics and Genomic Medicine 8(2): e1061.

DOI: http://dx.doi.org/10.1002/mgg3.1061

Louicharoen C. et al. (12 authors) (2009). Positively selected G6PD-Mahidol mutation reduces Plasmodium vivax density in Southeast Asians. Science 80(326): 1549-1564. DOI: https://doi.org/10.1126/science.1178849

Luzzatto L., Mehta A. \& Vulliamy T. (2011). Glucose-6phosphate dehydrogenase deficiency. In: The Online Metabolic and Molecular Bases of Inherited Diseases (eds. Valle D.L., Antonarakis S., Balliabio A., Beaudet A.L., Vogelsten B. \& Mitchell G.A.). McGrow Hill Global Education Holdings Inc., New York, USA. DOI: https://doi.org/10.1036/ommbid.212

Maiga B., Dolo A., Campino S., Sepulveda N., Corran P., Rockett K. A., Troye-Blomberg M., Doumbo O.K. \& 
Clark T.G. (2014). Glucose-6-phosphate dehydrogenase polymorphisms and susceptibility to mild malaria in Dogon and Fulani, Mali. Malaria Journal 13(1): 1-12.

DOI: https://doi.org/10.1186/1475-2875-13-270

Manjurano A. et al. (12 authors) (2015). African Glucose6-Phosphate Dehydrogenase Alleles Associated with Protection from Severe Malaria in Heterozygous Females in Tanzania. PLoS Genetics 11(2): 1-14.

DOI: https://doi.org/10.1371/journal.pgen.1004960

Niz M. De et al. (12 authors) (2013). Tools for mass screening of G6PD deficiency : validation of the WST8/1-methoxyPMS enzymatic assay in Uganda. Malaria Journal 12: 210 (2013).

DOI: https://doi.org/10.1186/1475-2875-12-210

Peng Q., Li S., Ma K., Li W., Ma Q., He X., He Y., He T. \& Lu X. (2015). Large cohort screening of G6PD deficiency and the mutational spectrum in the Dongguan District in Southern China. PloS One 10(3): e 0120683.

DOI: https://doi.org/10.1371/journal.pone.0120683
Rehman A., Shehadeh M., Khirfan D. \& Jones A. (2018). Severe acute haemolytic anaemia associated with severe methaemoglobinaemia in a G6PD-deficient man. BMJ Case Reports 2018: bcr2017223369.

DOI: https://doi.org/10.1136/bcr-2017-223369

Silva K.T. (2014). Decolonization, Development and Disease. A Social History of Malaria in Sri Lanka. Orient and Blackswan Pvt. Ltd., Hyderabad, India.

Tantular I.S. \& Kawamoto F. (2003). An improved, simple screening method for detection of glucose-6-phosphate dehydrogenase deficiency. Tropical Medicine and International Health 8(6): 569-574.

DOI: https://doi.org/10.1046/j.1365-3156.2003.01055.x

World Health Organization (2016). WHO certifies Sri Lanka malaria-free. Available at www.sero.who.int/mediacentre/ releases/2016/1631/en

Zerbino D.R. et al. (57 authors) (2018). Ensembl 2018. Nucleic Acids Research 46(D1): D754-D761.

DOI: https://doi.org/10.1093/nar/gkx1098 